\title{
Incidência de internações por eventos adversos a medicamentos em Minas Gerais
}

\author{
Incidence of hospitalizations due to adverse drug events in Minas Gerais, Brazil
}

Yasmin de Oliveira Machado Silva ${ }^{1}$, Marina Guimarães Lima² $₫$

${ }^{1}$ Curso de Farmácia da Universidade Federal de Minas Gerais (UFMG). Belo Horizonte, MG.

2 Departamento de Farmácia Social da Faculdade de Farmácia da UFMG. Belo Horizonte, MG.

\section{RESUMO}

Objetivos: Calcular a proporção de internações hospitalares pelo Sistema Único de Saúde no Estado de Minas Gerais decorrentes de eventos adversos a medicamentos, descrevendo-as segundo variáveis demográficas, tempo de permanência, especialidade médica e causa de internação. Métodos: Estudo transversal com dados secundários, obtidos do banco de dados do Sistema de Informações Hospitalares, referentes a internações hospitalares pagas pelo Sistema Único de Saúde em Minas Gerais no período de 2012 a 2014. A incidência de internações por eventos adversos a medicamentos foi calculada e expressa por 1.000 internações. Os totais de internações e de internações por eventos adversos a medicamentos foram descritos segundo o sexo e a faixa etária do paciente, o tempo de permanência no hospital e a especialidade médica. A comparação entre as frequências das diferentes categorias das variáveis em estudo foi realizada por meio do teste do qui-quadrado, empregando-se nível de significância de 5\%.

Resultados: A incidência de internações por eventos adversos a medicamentos foi de 25,9 por mil internações pagas pelo Sistema Único de Saúde. Os homens apresentaram maior proporção de internações por eventos adversos a medicamentos em comparação às mulheres $(2,7 \%$ contra 2,4\%). Dentre as faixas etárias, a que apresentou maior taxa de internações por eventos adversos a medicamentos foi a de 60 anos ou mais $(3,4 \%)$ e a menor taxa ocorreu nas crianças até 9 anos de idade $(0,6 \%)$. Foi observada uma maior proporção de hospitalizações por eventos adversos a medicamentos nas situações em que o tempo de permanência foi de 15 dias ou mais (7,6\%) e na especialidade médica de clínica psiquiátrica $(8,3 \%)$.

Conclusões: Os eventos adversos a medicamentos constituíram uma importante causa de internação hospitalar no SUS em Minas Gerais entre 2012 e 2014. A ocorrência de internação por eventos adversos a medicamentos apresentou associação com sexo masculino, faixa etária de 60 anos ou mais, maior tempo de permanência no hospital e especialidade médica de clínica psiquiátrica. Ações de fomento da farmacovigilância nas instituições de saúde são necessárias para a prevenção de eventos adversos a medicamentos.

DESCRITORES: vigilância em saúde pública; farmacovigilância; efeitos colaterais e reações adversas relacionados a medicamentos.

\section{ABSTRACT}

Aims: To estimate the rate of hospital admissions due to adverse drug events under the Unified Public Health System in the State of Minas Gerais, Brazil, according to demographic variables, length of stay, medical specialty, and cause of hospitalization.

Methods: Cross-sectional study with secondary data on hospital admissions paid for under the Unified Health System in Minas Gerais between 2012 and 2014. The incidence of hospitalizations for adverse drug events was calculated and expressed per 1,000 admissions. The total number of admissions and of hospitalizations for adverse drug events was described according to the patient's gender and age, length of hospital stay, and the medical specialty. The frequencies of the different categories of the variables of interest were compared using the chi-square test and a 5\% significance level.

Results: The incidence of hospitalizations for adverse drug events was 25.9 per 1,000 admissions paid for by the Unified Public Health System. Men had a higher rate of hospitalizations for adverse drug events than women $(2.7 \%$ vs. $2.4 \%)$. In terms of age, the highest rate of hospitalizations for adverse drug events was observed among individuals aged 60 years or older (3.4\%) and the lowest rate was among children aged up to 9 years $(0.6 \%)$. A higher rate of hospitalizations for adverse drug events was observed when the length of stay was 15 days or longer $(7.6 \%)$ and in patients admitted from the psychiatric service $(8.3 \%)$.

Conclusions: Adverse drug events were an important cause of hospitalization under the Unified Public Health System in Minas Gerais between 2012 and 2014. Hospitalization for adverse drug events was associated with being male, being 60 years or older, having a longer hospital stay, and being admitted from the psychiatric service. Actions that promote pharmacovigilance in health institutions are necessary for the prevention of adverse drug events.

KEY WORDS: public health surveillance; pharmacovigilance; drug-related side effects and adverse reactions. 
Abreviaturas: SUS, Sistema Único de Saúde; CID-10, 10aㅡ Classificação Internacional de Doenças; DATASUS, Departamento de Informática do Sistema Único de Saúde; EAM, eventos adversos a medicamentos.

\section{INTRODUÇÃO}

Medicamentos representam a tecnologia sanitária mais empregada no cuidado aos pacientes nos diferentes níveis de atenção à saúde [1]. Assim como acontece com outras tecnologias, a incidência significativa de eventos adversos a medicamentos (EAM) pode ser explicada pelo fato de os medicamentos apresentarem riscos inerentes ao perfil de segurança, sendo necessário o acompanhamento de sua utilização para identificação de riscos, controle e prevenção dos problemas [2].

Os estudos atuais sobre eventos adversos de diversos tipos de terapia buscam compreender sua importância e o impacto na saúde pública. A margem da incidência de EAM, na literatura, é de 6,5\% dos pacientes adultos internados e $27,4 \%$ dos pacientes ambulatoriais, enquanto que revisões sistemáticas apontam uma margem de $5 \%$ a $8 \%$ de EAM como causa de internações hospitalares, com consequente aumento do tempo de internação, de recursos materiais e profissionais, e consumo substancial dos recursos da saúde [3].

No estado do Rio de Janeiro, por meio de uma análise dos dados do Sistema de Informações Hospitalares, entre os anos 1999 e 2002, a taxa de internação tendo como causa EAM foi de 1,8 a cada mil internações, considerando o diagnóstico principal e o secundário [4]. A taxa de incidência de EAM aumentou de 1,4/1000 no ano de 1999 para 2,3/1000 no ano de 2002, sendo encontrado maior número de casos entre os homens $(64,5 \%)$, em hospitais contratados $(34,9 \%)$, e com $84,1 \%$ com desfecho de alta hospitalar. Já uma revisão sistemática de Varallo et al. [5] identificou maior número de casos entre mulheres $(66,1 \%)$.

Ao considerar o impacto de uma doença na população, devem-se contabilizar os EAM e o que é gasto para identificá-los, tratá-los e preveni-los, uma vez que os medicamentos são itens relevantes para orçamento e estratégias de saúde. Estudos como o de Mastroianni et al. [6] demonstram a relação entre a quantidade de medicamentos utilizados e a probabilidade de ocorrer internação hospitalar por EAM, e alerta que até dois terços dessas internações podem resultar de ineficácia terapêutica ou adesão inadequada ao tratamento [6]. Outro dado relevante das internações hospitalares por EAM é a estimativa de que 3,8\% delas são oriundas de interações medicamentosas [7], o que também se pode relacionar à quantidade de medicamentos utilizados antes da internação. Portanto, conhecer a população e o perfil das internações por causa de EAM é uma importante informação para a saúde pública.

O presente trabalho teve como objetivo calcular a proporção de internações hospitalares pelo Sistema Único de Saúde (SUS) decorrentes de EAM, no estado de Minas Gerais, no período de 2012 a 2014, descrevendo essas internações segundo variáveis demográficas e de serviços de saúde.

\section{MÉTODOS}

Foi realizado um estudo transversal com dados secundários de internações hospitalares contidos no banco de dados do Sistema de Informações Hospitalares disponível no Departamento de Informática do Sistema Único de Saúde (DATASUS). Dentre os dados disponíveis, foram utilizados aqueles que constam nas Autorizações de Internação Hospitalar, que são informações obrigatórias preenchidas pelas instituições de saúde e que, após análise pelo Ministério da Saúde, validam o consequente pagamento pelos serviços prestados à população brasileira. Os dados foram tabulados e tratados com a utilização do programa Tabwin, disponível para download na página eletrônica do DATASUS.

A amostra do estudo foi extraída dos dados disponíveis nas autorizações de internação hospitalar, de 2012 a 2014, em estabelecimentos de saúde pagos pelo SUS, de natureza jurídica municipal, estadual, universitária, filantrópica e privado/contratado, dentro do estado de Minas Gerais. Os desfechos considerados foram o "diagnóstico principal" e o "diagnóstico secundário", utilizando para a classificação de casos de internações por EAM os códigos da $10^{\underline{a}}$ Classificação Internacional de Doenças (CID-10), segundo metodologia adotada por Rozenfeld [4]. A principal categoria foi "efeitos adversos de drogas, medicamentos e substâncias biológicas usadas com finalidade terapêutica", e os demais códigos relacionados ao assunto foram "intoxicações e sequelas de intoxicações"; "erros na prescrição ou na administração"; "diluição ou dosagem"; "lesões autoprovocadas intencionalmente"; "envenenamentos cuja intenção é indeterminada"; "envenenamentos acidentais"; "complicações consequentes à infusão, transfusão ou injeção terapêutica"; e "doenças cuja causa externa possibilita relacionar fármacos como responsáveis". Essas inclusões abrangem o estudo para 
os demais agravos provocados por EAM. A Tabela 1 indica os códigos da CID-10 selecionados no campo de desfecho.

Tabela 1. Campos diagnósticos selecionados a partir da Classificação Internacional de Doenças (CID-10) como causa de internações hospitalares por eventos adversos a medicamentos, no estado de Minas Gerais, nos anos de 2012 a 2014.

\begin{tabular}{|c|c|}
\hline Capítulo & Código \\
\hline $\begin{array}{l}\text { III - Doenças do sangue e dos órgãos } \\
\text { hematopoiéticos e alguns transtornos } \\
\text { imunitários }\end{array}$ & $\begin{array}{l}\text { D52.1, D59.0, D59.2, D61.1, } \\
\text { D68.3, D69.5, D70, D74.8 }\end{array}$ \\
\hline $\begin{array}{l}\text { IV - Doenças nutricionais, endócrinas e } \\
\text { metabólicas }\end{array}$ & $\begin{array}{l}\text { E03.2, E06.4, E10-E14, E15, E16.0, } \\
\text { E22.1, E23.1, E24.2, E27.3, E28.0, } \\
\text { E28.1, E29.1, E66.1 }\end{array}$ \\
\hline V - Transtornos mentais e comportamentais & F11, F13, F19, F55 \\
\hline $\mathrm{VI}$ - Doenças do sistema nervoso & $\begin{array}{l}\text { G21.0, G21.1, G24.0, G25.1, } \\
\text { G25.3, G25.4, G25.6, G40.5, } \\
\text { G43.0, G43.1, G43.2, G43.3, } \\
\text { G43.8, G43.9, G44.4, G62.0, } \\
\text { G70.0, G71.1, G72.0, G93.7 }\end{array}$ \\
\hline VII - Doenças do olho e anexos & $\mathrm{H} 18.0, \mathrm{H} 26.3, \mathrm{H} 40.6$ \\
\hline $\begin{array}{l}\text { VIII - Doenças do ouvido e da apófise } \\
\text { mastoide }\end{array}$ & H91.0 \\
\hline IX - Doenças do aparelho circulatório & $\begin{array}{l}142.7,180,180.0,180.01,180.2 \\
180.3,180.8,180.9,195.2\end{array}$ \\
\hline X - Doenças do aparelho circulatório & $\mathrm{J} 70.2, \mathrm{~J} 70.3, \mathrm{~J} 70.4$ \\
\hline XI - Doenças do aparelho digestivo & K25, K26, K71 \\
\hline $\begin{array}{l}\text { XII - Doenças da pele e do tecido } \\
\text { subcutâneo }\end{array}$ & $\begin{array}{l}\text { L10.5, L23.3, L24.4, L25.1, L27.0, } \\
\text { L27.1, L43.2, L56.0, L56.1, L64.0, } \\
\text { L65, L68.0, L68.1, L71.0, L93 }\end{array}$ \\
\hline $\begin{array}{l}\text { XIII - Doenças do tecido osteomuscular e } \\
\text { do tecido conjuntivo }\end{array}$ & $\begin{array}{l}\text { M10.2, M32.0, M34.2, M80.4, } \\
\text { M81.4, M83.5, M87.1 }\end{array}$ \\
\hline XIV - Doenças do aparelho genitourinário & N14.0, N14.1, N14.2 \\
\hline $\begin{array}{l}\text { XVIII - Sintomas, sinais e achados anormais } \\
\text { de exames clínicos e de laboratório não } \\
\text { classificados em outra parte }\end{array}$ & $\begin{array}{l}\text { R00.1, R78.1, R78.4, R78.5, R78.6, } \\
\text { R78.8, R82.5 }\end{array}$ \\
\hline $\begin{array}{l}\text { XIX - Lesões, envenenamentos, e algumas } \\
\text { outras consequências de causas externas }\end{array}$ & $\begin{array}{l}\text { T36 - T39, T40.2, T40.3, T40.4, } \\
\text { T41 - T50, T80, T88.2, T88.3, } \\
\text { T88.5, T88.6, T88.7, T96 }\end{array}$ \\
\hline $\begin{array}{l}X X \text { - Causas externas de morbidade e de } \\
\text { mortalidade }\end{array}$ & $\begin{array}{l}X 40, X 41, X 43, X 60, X 61, X 63, Y 10, \\
Y 11, Y 13, Y 40-Y 57, Y 63.0, Y 63.1 \\
\text { Y63.8, Y63.9, Y65.0, Y65.1 }\end{array}$ \\
\hline
\end{tabular}

Por meio do programa Tabwin, foram extraídos dados referentes ao número de internações e ao valor financeiro pago pelo SUS no Estado de Minas Gerais. A análise de dados incluiu todos os dados referentes às internações, não havendo perda de informação. A incidência de internações por EAM foi calculada para cada ano do período de estudo dividindo-se o número de internações por EAM pelo número total de internações. As incidências foram expressas por 1.000 internações. O número total de internações e de internações por EAM no período de 2012 a 2014 foram descritos segundo o sexo e a faixa etária do paciente, o tempo de permanência no hospital e a especialidade médica. As internações por EAM, adicionalmente, foram descritas segundo o CID-10. A comparação entre as frequências de internações por EAM das diferentes categorias de sexo, faixa etária, tempo de internação e especialidade médica foi realizada por meio do teste do qui-quadrado, empregando-se nível de significância de $5 \%$.

O estudo utilizou informações disponíveis ao público no site DATASUS, cujos dados são apresentados de forma agregada e não individual. Devido a este fato, o projeto de pesquisa não foi submetido a um Comitê de Ética em Pesquisa.

\section{RESULTADOS}

Entre os anos 2012 a 2014 ocorreram no Estado de Minas Gerais 3.628.080 internações hospitalares pagas pelo SUS, cuja média do valor financeiro por internação foi de $\mathrm{R} \$ 1.262,19$. O número de internações que tiveram como causa os EAM foi de 93.470, representando uma taxa de 25,8/1.000 internações pagas pelo SUS. O valor médio das internações por EAM foi de R $\$ 732,53$. O número de internações por EAM foi de 30.849 em 2012, 31.519 em 2013 e 31.102 em 2014. O ano de 2014 teve a menor incidência observada, 25,0 casos em mil internações, enquanto que os anos de 2012 e 2013 tiveram respectivamente 26,1 e 26,2 casos a cada mil internações.

As características das internações gerais e por EAM podem ser vistas na Tabela 2. As especialidades com maior frequência de internações gerais, segundo a classificação adotada pelo DATASUS, foram "Outras", que incluem cirurgia, obstetrícia, doença crônica, pneumologia sanitária, reabilitação e leito dia (cirurgia, aids, intercorrência pós transplante, saúde mental e não discriminado). Diferentemente das internações gerais, a especialidade com mais internações por EAM foi a clínica médica. Os homens apresentaram maior proporção de internações por EAM em comparação às mulheres. Dentre as faixas etárias, a que apresentou maior frequência de internações por EAM foi a de 60 anos ou mais e a com menor frequência foi a de até nove anos de idade. Foi observada maior proporção de hospitalizações por EAM nas situações em que o tempo de permanência foi de 15 dias ou mais e na especialidade médica de clínica psiquiátrica.

Também foi investigada a frequência de internações por EAM de acordo com a classificação de diagnósticos CID-10. A classificação mais frequente foi a composta por doenças nutricionais, endócrinas e metabólicas, com $54,2 \%$, seguida das doenças do aparelho circulatório, com 20,3\% (Tabela 3). 
Tabela 2. Características das internações gerais e por eventos adversos a medicamentos pagas pelo SUS no Estado de Minas Gerais, 2012 a 2014

\begin{tabular}{|c|c|c|c|c|c|c|}
\hline \multirow{2}{*}{ Variável } & \multicolumn{2}{|c|}{ Internações gerais* } & \multicolumn{2}{|c|}{ Internações por EAM** } & \multirow{2}{*}{$\begin{array}{c}\text { Total de } \\
\text { internaçôes }\end{array}$} & \multirow{2}{*}{$\mathrm{p}^{\dagger}$} \\
\hline & $\mathbf{n}$ & $\%$ & n & $\%$ & & \\
\hline \multicolumn{7}{|l|}{ Sexo } \\
\hline Masculino & 1.511 .566 & 97,2 & 43.322 & 2,8 & 1.554 .888 & \multirow{2}{*}{$<0,001$} \\
\hline Feminino & 2.023 .044 & 97,6 & 50.148 & 2,4 & 2.073 .192 & \\
\hline \multicolumn{7}{|l|}{ Faixa etária } \\
\hline 0 a 09 anos & 392.323 & 99,4 & 2.310 & 0,6 & 394.633 & \multirow{4}{*}{$<0,001$} \\
\hline 10 a 19 anos & 306.451 & 98,4 & 5.008 & 1,6 & 311.459 & \\
\hline 20 a 59 anos & 1.850 .009 & 97,4 & 49.957 & 2,6 & 1.899 .966 & \\
\hline 60 anos ou mais & 985.827 & 96,5 & 36.195 & 3,5 & 1.022 .022 & \\
\hline \multicolumn{7}{|c|}{ Tempo de permanência } \\
\hline 0 a 1 dia & 847.579 & 99,1 & 7.471 & 0,9 & 855.050 & \multirow{3}{*}{$<0,001$} \\
\hline 2 a 14 dias & 2.405 .907 & 97,5 & 60.873 & 2,5 & 2.466 .780 & \\
\hline 15 dias ou mais & 281.124 & 91,8 & 25.126 & 8,2 & 306.250 & \\
\hline \multicolumn{7}{|l|}{ Especialidade médica } \\
\hline Clínica médica & 1.343 .513 & 94,8 & 74.246 & 5,2 & 1.417 .759 & \multirow{4}{*}{$<0,001$} \\
\hline Clínica psiquiátrica & 80.742 & 91,0 & 8.014 & 9,0 & 88.756 & \\
\hline Pediatria & 303.312 & 99,0 & 2.944 & 1,0 & 306.256 & \\
\hline Outras & 1.807 .043 & 99,5 & 8.266 & 0,5 & 1.815 .309 & \\
\hline Total & 3.534 .610 & & 93.470 & & 3.628 .080 & \\
\hline
\end{tabular}

* O número de internações gerais foi calculado subtraindo-se o número de internações por EAM do número total de internações.

** EAM:eventos adversos a medicamentos.

† Qui-quadrado.

Tabela 3. Frequência da classificação de internações hospitalares por eventos adversos a medicamentos de acordo com a Classificação Internacional de Doenças (CID-10) no estado de Minas Gerais, 2012 a 2014.

\begin{tabular}{|c|c|c|}
\hline \multirow{2}{*}{ Classificação CID-10 } & \multicolumn{2}{|c|}{ Frequência } \\
\hline & $\mathbf{n}$ & $\%$ \\
\hline III - Doenças do sangue e dos órgãos hematopoiéticos e alguns transtornos imunitários & 446 & 0,5 \\
\hline IV - Doenças nutricionais, endócrinas e metabólicas & 50.671 & 54,2 \\
\hline V - Transtornos mentais e comportamentais & 9.186 & 9,8 \\
\hline $\mathrm{VI}$ - Doenças do sistema nervoso & 2.073 & 2,3 \\
\hline VII - Doenças do olho e anexos & 11 & 0,0 \\
\hline VIII - Doenças do ouvido e da apófise mastoide & 0 & 0 \\
\hline IX - Doenças do aparelho circulatório & 18.975 & 20,3 \\
\hline$X$ - Doenças do aparelho circulatório & 0 & 0 \\
\hline XI - Doenças do aparelho digestivo & 2.903 & 3,1 \\
\hline XII - Doenças da pele e do tecido subcutâneo & 1.411 & 1,5 \\
\hline XIII - Doenças do tecido osteomuscular e do tecido conjuntivo & 14 & 0,0 \\
\hline XIV - Doenças do aparelho genitourinário & 17 & 0,0 \\
\hline XVIII - Sintomas, sinais e achados anormais de exames clínicos e de laboratório não classificados em outra parte & 59 & 0,1 \\
\hline XIX - Lesões, envenenamentos, e algumas outras consequências de causas externas & 5.108 & 5,5 \\
\hline XX - Causas externas de morbidade e de mortalidade & 2.594 & 2,7 \\
\hline Total & 93.468 & 100,0 \\
\hline
\end{tabular}




\section{DISCUSSÃO}

Muitos estudos sobre os EAM abordam o tema sob a perspectiva de um agravo durante a assistência hospitalar ou ambulatorial, ocorrendo em $3 \%$ a $18,3 \%$ do total de internações [6-9], mas não como causa de internações. Alguns estudos que investigaram as internações hospitalares tendo como motivo o uso prévio de medicamentos estimam uma variação de $0,5 \%$ a $32,9 \%$, cuja diferença pode ser relacionada ao desenho do estudo, como a população amostral, os métodos de coleta e a seleção de dados [5]. Neste estudo, com dados do estado de Minas Gerais entre 2012 e 2014, o percentual de internações tendo como causa os EAM está compreendido na variação que consta na literatura, sendo uma proporção relativamente elevada.

A população com 60 anos ou mais, no presente estudo, apresentou maior proporção de internações por EAM em comparação com as outras faixas etárias. Paula et al. [10], ao analisarem a população brasileira idosa e as internações decorrentes do uso de medicamentos, concluíram que os EAM representam uma causa importante de internações hospitalares nessa faixa etária e vem aumentando no decorrer do tempo, chegando a quase $14 \%$ de aumento em quatro anos. Os idosos consomem mais medicamentos devido ao acúmulo das comorbidades e aumento da frequência das doenças crônicas, que muitas vezes necessitam de medicação contínua [11,12]. Mastroianni et al. [6], com um estudo abrangente de faixa etária, relacionaram a polifarmácia como fator significativo do aumento de internações por EAM na população como um todo.

Embora o maior número de casos de EAM tenha ocorrido no sexo feminino, a maior taxa de internações por EAM ocorreu nos pacientes do sexo masculino, quando se considera o número total de internações para cada sexo. A diferença, embora estatisticamente significativa, foi pequena, podendo ser considerada clinicamente irrelevante. Estudos publicados na literatura científica $[8,11]$ não identificaram o sexo como fator de risco para EAM.

No presente estudo observou-se maior proporção de internações por EAM entre os indivíduos com maior tempo de permanência hospitalar. Segundo um estudo realizado em um hospital no Brasil, há associação positiva entre tempo de permanência e a ocorrência de EAM, o que demanda um monitoramento mais frequente dos pacientes com maior tempo de internação por parte da equipe de saúde [11].

A especialidade médica com maior proporção de internações por EAM no período em estudo no estado de Minas Gerais foi a clínica psiquiátrica. Rozenfeld [4] encontrou como especialidade médica mais frequente a psiquiatria entre os agravos provocados por medicamentos nas Autorizações de Internações Hospitalares do SUS no estado do Rio de Janeiro.

O presente estudo apresentou limitações. Devido ao problema da subnotificação pela equipe de saúde, os dados coletados podem estar subestimados, sendo possível que as internações por EAM no estado de Minas Gerais tenham uma incidência maior. Outra limitação observada foi a impossibilidade de coletar a incidência e frequência dos medicamentos envolvidos nos eventos adversos. Entretanto, este estudo apresenta dados pouco explorados na literatura, que em geral aborda os EAM como consequência de uma internação hospitalar, e não como causa.

A notificação dos EAM é fundamental para subsidiar ações de segurança do paciente destinadas a prevenir danos. As ações de farmacovigilância no Brasil são recentes, com legislações específicas a partir de 1976, mas têm evoluído. Além dos vários tipos de instituições colaboradoras para informações de farmacovigilância, equipes multidisciplinares e órgãos responsáveis, a grande evolução na atualidade foi incluir o usuário do medicamento como parte fundamental do processo de tratamento, sendo ele o objeto central do cuidado. A propensão é alinhar e compartilhar informações não somente obtidas no Brasil, mas internacionalmente, como as estratégias em andamento nos países desenvolvidos [13].

Podemos concluir que os EAM constituíram uma importante causa de internação hospitalar no SUS em Minas Gerais entre 2012 e 2014. Recomenda-se a capacitação das equipes de saúde em detectar EAM, com disponibilização de um meio prático que permita notificações voluntárias pelos funcionários e ações de farmacovigilância nas instituições de saúde. Também é relevante o aumento da resolutividade da Atenção Primária à Saúde para prevenir EAM e promover o uso racional da farmacoterapia.

\section{NOTAS}

Apoio financeiro

Este estudo não recebeu apoio financeiro de fontes externas.

Declaração de conflito de interesses

As autoras declaram não haver conflitos de interesses relevantes ao conteúdo deste estudo. Elas informam ter tido acesso a todos os dados obtidos e assumem completa responsabilidade pela integridade dos resultados. 


\section{REFERÊNCIAS}

1. Carvalho WS, Moreira AM, Magalhães SMS. Eventos adversos a medicamentos. In: Acurcio FA, organizador. Medicamentos: políticas, assistência farmacêutica, farmacoepidemiologia e farmacoeconomia. Belo Horizonte: COOPMED; 2013. p. 147-78.

2. Organização Mundial da Saúde. Departamento de Medicamentos Essenciais e Outros Medicamentos. A importância da farmacovigilância: monitorização da segurança dos medicamentos. Brasília: Organização Pan-Americana da Saúde; 2005.

3. Capucho HC, Carvalho FD, Cassiani SHB. Farmacovigilância: gerenciamento de riscos da terapia medicamentosa para a segurança do paciente. São Paulo: Yendis; 2012.

4. Rozenfeld S. Agravos provocados por medicamentos em hospitais do Estado do Rio de Janeiro, Brasil. Rev Saúde Pública. 2007;41(1):108-15. https://doi.org/10.1590/S0034-89102006005000012

5. Varallo FR, Guimarães SOP, Abjaude SAR, Mastroianni PC. Causes for the underreporting of adverse drug events by health professionals: a systematic review. Rev Esc Enferm USP. 2014;48(4):739-47. https://doi.org/10.1590/S0080623420140000400023

6. Mastroianni PC, Varallo FR, Barg MS, Noto AR, Galduróz JCF. Contribuição do uso de medicamentos para a admissão hospitalar. Braz J Pharm Sci. 2009;45(1):163-70. https://doi.org/10.1590/S1984-82502009000100020

7. Varallo FR, Costa MA, Mastroianni PC. Potenciais interações medicamentosas responsáveis por internações hospitalares. Rev Ciênc Farm Básica Apl. 2013;34(1):79-85.

8. Lobo MGAA, Pinheiro SMB, Castro JGD, Momenté VG, Pranchevicius M-CS. Adverse drug reaction monitoring: support for pharmacovigilance at a tertiary care hospital in Northern Brazil. BMC Pharmacol Toxicol. 2013;14:5. https:// doi.org/10.1186/2050-6511-14-5

9. Porto S, Martins M, Mendes W, Travassos C. A magnitude financeira dos eventos adversos em hospitais no Brasil. Rev Port Saúde Pública. 2010; Vol Temat (10):74-80.

10. Paula TC, Bochner R, Montilla DER. Análise clínica e epidemiológica das internações hospitalares de idosos decorrentes de intoxicações e efeitos adversos de medicamentos, Brasil, de 2004 a 2008. Rev Bras Epidemiol. 2012;15(4):828-44. https://doi.org/10.1590/S1415-790X2012000400014

11. Giordani F, Rozenfeld S, Martins M. Adverse drug events identified by triggers at a teaching hospital in Brazil. BMC Pharmacol Toxicol. 2014;15:71. https://doi.org/10.1186/2050-6511-15-71

12. Mendes W, Martins M, Rozenfeld S, Travassos C. The assessment of adverse events in hospitals in Brazil. Int J Qual Health Care. 2009;21(4):279-84. https://doi.org/10.1093/intqhe/mzp022

13. Mastroianni PC, Varallo FR, Hernandes CD. Brazilian regulation in pharmacovigilance: a review. Pharm Regul Aff. 2016;5:164. 